\title{
Allopatric speciation rather than ecological speciation drives evolution in an Amazonian fish.
}

\author{
Nicolas Leroux ${ }^{1}$, François-Étienne Sylvain ${ }^{1}$, Eric Normandeau ${ }^{1}$, Aleicia Holland ${ }^{2}$, \\ Adalberto Luis $\mathrm{Val}^{3}$, and Nicolas Derome ${ }^{1}$ \\ ${ }^{1}$ Universite Laval Institut de Biologie Integrative et des Systemes \\ ${ }^{2}$ La Trobe University School of Life Sciences \\ ${ }^{3}$ Instituto Nacional de Pesquisas da Amazonia
}

February 2, 2022

\begin{abstract}
Amazonia is characterized by very heterogeneous riverscapes dominated by two drastically divergent water types: black (ionpoor, dissolved organic carbonate rich and acidic) and white (nutrient rich and turbid) waters. Recent phylogeographic and genomic studies have associated the ecotone formed by these environments to ecologically driven speciation in fishes. With the objective of better understanding the evolutionary forces behind the Amazonian Teleostean diversification, we sampled 240 Mesonauta festivus from 12 sites on a wide area of the Amazonian basin. These sites included three confluences of black and white water environments to seek for repeated evidences of ecological speciation at these ecotones. Results obtained through our genetic assessment based on 41,268 SNPs contrast with previous findings and supports a low structuring power of water types. Conversely, we detected a strong pattern of isolation by unidirectional downstream water current and evidence of past events of vicariance potentially linked to the Amazon River formation. Using a combination of population genetic, phylogeographic analysis and environmental association models, we decomposed the spatial variance from the environmental genetic variance specifically to assess which evolutive forces have shaped inter-population differences in $M$. festivus' genome. Our sampling design comprising four major Amazonian rivers and three confluences of black and white water rivers supports the possibility that past studies potentially confounded ecological speciation with a site effect unrepresentative of the full Amazonian watershed. While ecological speciation admittedly played a role in Amazonian fish species diversification, we argue that neutral evolutionary processes explain most of the divergence between $M$. festivus populations.
\end{abstract}

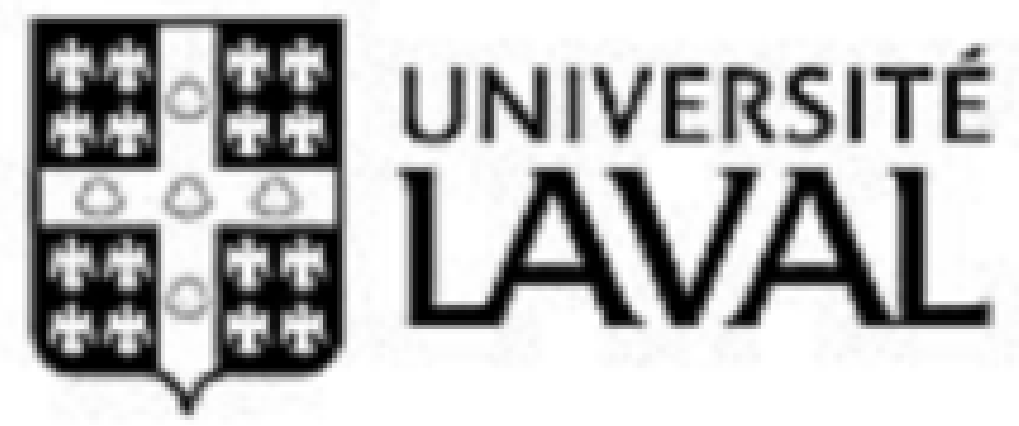




\section{Introduction}

The neotropical region hosts the most diverse freshwater fish fauna in the world, with estimates varying from 6,000 to 8,000 fish species (Reis 2013). While knowledge about its diversity is central for its conservation (Beheregaray et al. 2015), this megadiverse region is understudied and studies describing the evolutionary history of South American fish are still scarce. Multiple speciation hypotheses have been constructed based on a mix of geologic, phenotypic and genetic data (Hubert et al. 2007, Ríos-Villamizar et al. 2013, Bragança and Costa 2018). From these, ecological speciation, the evolution of new species from populations affected by an ecologically driven divergent natural selection (Rundle \& Nosil 2005; Schluter 2001; 2009), allopatric speciation, and the evolution of new species by vicariance events leading to the accumulation of mutations (Yamaguchi \& Iwasa 2013), are promising hypotheses.

The first hypothesis, ecological speciation, suggests that speciation of Amazonian fish species is based on the presence of very heterogeneous Amazonian aquatic ecosystems resulting from two water types with contrasting chemistry. White water has a cloudy appearance caused by a large amount of silicate in suspension. Also, it has a lower amount of dissolved organic carbonate, a circumneutral $\mathrm{pH}$ and contains a higher quantity of ions than black water which is rich in DOC, acidic and ion-poor. For instance, conductivity at $20^{\circ} \mathrm{C}$ for black water is close to $8 \mu \mathrm{S} / \mathrm{cm}$ versus $70 \mu \mathrm{S} / \mathrm{cm}$ for white water. In the same way, the $\mathrm{pH}$ for black water is usually lower than five, compared to seven for white water (Sioli 1984, Val and Almeida-Val 1995, Ríos-Villamizar et al. 2013, Holland et al. 2017). These variable parameters could represent physiological challenges for fish species (Ríos-Villamizar et al. 2013). The high dissimilarity of the two water types could lead to natural selection against migrants, a post-zygotic isolation which could reduce gene flow between white and black water sites (Rundle and Nosil 2005). Additionally, the different selective pressures in each environment could lead to directional and discrete modifications in small and specific regions of the genome (Schluter 2001, Rundle and Nosil 2005).

On one hand, the Amazonian ecological speciation hypothesis is mainly based on recent genetic studies conducted at the confluence of the Amazon's main tributaries, the Rio Negro (black water) and the Rio Solimões (white water) (Cooke et al. 2012; 2012b; 2014; Beheregaray et al. 2015). For instance, Beheregaray et al. (2015) detected strong natural selection with gene flow based on the observation of a strong neutral genetic divergence between Triportheus albus populations living in divergent Amazonian water types. Also, a genome scan analysis (Cooke et al. 2012a) and a reproductive isolation experiment (Pires et al. 2018) have suggested that multiple fish species from these same two major rivers have a strong genetic divergence as a result of possible ecological speciation associated with the waters contrasting environmental conditions. The conclusions of these studies tend to disagree with the well-established hypothesis of evolution of Amazonian fish by allopatry (Abreu et al. 2020; Roxo et al. 2012). However, the sampling designs of these papers (Cooke et al. 2012; 2012b; 2014; Beheregaray et al. 2015) are all very similar. They all sampled the same confluence of rivers which could lead to repeated misinterpretations of results based on a site-specific effect rather than on an ecological process representative of the full Amazonian watershed (Smart 2017).

On the other hand, speciation by allopatry is a much older and less speculative evolutionary hypothesis based on speciation events having occurred in lakes or rivers isolated by geologic and sea-level fluctuation events since the Pliocene (Hubert \& Renno 2006). Typically, this evolutionary hypothesis assumes that isolated populations are evolving independently by genetic drift, leading to genome wide random variations of allele frequencies in separated populations. Also, this genetic divergence is linear with time, meaning that populations separated by older geologic processes should be increasingly different. The Amazon has a complex but well-studied geologic past which has been the source of very informative and robust evolutionary hypotheses (Albert et al. 2018; Hoorn et al. 2010; Potter 1997). Geologic studies revealed that the Amazonian region was characterized by a series of connected mega-lakes from the late Miocene until $~ 2.5$ Mya (Campbell et al. 2006). The modern Amazon formed after the breaching of the eastern rim of the sedimentary basin resulting from the Andean uplift, which led to the now-established connection with the Atlantic Ocean (Campbell et al. 2006). The water drainage engendered strong erosion and sediment accumulations, leading to the formation of thousands of small lakes and rivers (Moreira et al. 2020). These geological records are 
strongly supported by terrestrial diversification processes (Lynch Alfaro et al. 2015; Ribas et al. 2012) and coincide with important sea-level fluctuation events caused by the interglacial cycles of the last 2.75 Ma, leading to temporary salt-water incursions (Berger \& Loutre 2010; Grant et al. 2019). Likewise, sedimentation and saltwater incursions are natural processes that can cause isolation of freshwater bodies, a potential vector of evolution by allopatry (Lovejoy et al. 2006; Latrubesse \& Franzinelli 2005). Consequently, events of speciation by allopatry were previously linked to the Amazon formation and these interglacial cycles using mitochondrial molecular clock estimates in several fish species, i.e., Discus (Symphysodon sp.) (Farias \& Hrbek 2008), Steindachnerina sp. (Venere et al. 2008) and the miniature killifish (Fluviphylax) (Bragança \& Costa 2018).

Differentiating the effects of ecological speciation and allopatry is a complex challenge in the Amazon since its water chemistry is naturally linked to its geologic past. In this sense, the transition from black to white water (and vice versa) always takes origin from a geologic event that shaped today's watercourses. To decipher each evolutionary force effect, it is necessary to compare multiple black and white water boundaries and look for repeated evidence of ecological speciation. In the presence of ecological speciation mediated by water type, the similar environmental shift between the populations should lead to a directional genetic signal located on specific loci under selection and/or some genome-wide variations in allele frequency caused by the reduced gene flow between different water types (Rundle and Nosil 2005). On the contrary, evolution by allopatry should show a time-related linear genetic divergence, where populations who were separated by an older geologic process are more strongly differentiated genome wide. While selected loci tend to coincide with habitat variations, neutral loci mostly reflect the geographic connectivity between sites (Bierne et al. 2013), requiring the independent analysis of both types of loci independently to detect or deny a scenario of ecological speciation. It is also difficult to distinguish the molecular signatures of ecological speciation and allopatric evolution since the Amazonian watershed connectivity varied through past climate oscillations, saltwater incursions and geologic events (Nores 2004, Thom et al. 2020). Additionally, the high connectivity between rivers facilitates the migration and tends to weaken the genetic signal coming from older geologic processes.

Our study aimed to test the validity of the two different speciation hypotheses (ecological or allopatric) in relation to populations of Mesonauta festivus (Heckel 1840), one of the most widespread Teleosteans in the Amazon basin (Pires et al. 2015). Mesonauta festivus is a small cichlid ubiquitous to the Amazon basin. With its sedentary behaviour and limited long-distance migration ability, M. festivus has a very low upstream migration potential but a moderate downstream migration potential since it can let itself be carried by the current (Pires et al. 2015). Additionally, M. festivus shows strong parental care investment and a high environmental tolerance, allowing the species to live under different abiotic conditions, potentially facilitating the establishment of new populations (Pires et al. 2015). Here, we used a sampling design consisting of 12 sites spread throughout the Amazon basin and comprising three black and white water river confluences to (1) trace the phylogeographic history of $M$. festivus in the Amazonian watershed; (2) describe the importance of the diverging water characteristics on the genotype of M. festivus and (3) assess which scenario, either ecological speciation and/or allopatric speciation, better explains the genetic structure of $M$. festivus within the Amazon basin. Our approach based on a combination of population genetic, phylogeographic analysis and environmental association models helped us decipher the impact of the water's physicochemical characteristics and historical vicariance events as structuring factors for populations of $M$. festivus across the Amazonian watershed.

\section{Material and methods}

\section{Experimental design}

We sampled 240 M. festivus at 12 sites distributed across three major Amazonian rivers (i.e., Rio Branco, Rio Negro and Rio Solimões) [Fig. 1]. These sites comprise ecosystems with drastically divergent physicochemical 
parameters: five black water sites (BAR, NEG, CEM, ANA and TEF) and seven white water sites (PIR, SOL, MAN, JAR, JAC, CAT, BRA) [Table 1]. These sites comprise three confluence zones between black and white water sites : (1) Six sampling sites were close to the confluence of two major Amazonian tributaries, the Rio Negro and Rio Solimões (ANA, CEM, CAT, JAC, JAR and MAN); (2) three sites were close to the confluence of the Rio Branco and Rio Negro (BAR, NEG and BRA) and (3) three sites were close to the confluence of the Rio Tefé and Rio Solimões (TEF, SOL and PIR) [Fig. 1].

Field trips were conducted from September to December 2018-2019 during the dry season. Sites coordinates were recorded using the Global Positioning System (GPS) and the fishing perimeters were assessed in the field according to each site's local geography. We estimated the watercourse distance separating each sampling site using Google Earth pro (Image Landsat / Copernicus, U.S). A multiparameter YSI Professional+Series meter (YSI Inc/Xylem Inc USA) was used to characterize water physicochemical properties (conductivity and $\mathrm{pH}$ ) on sites. Two litres of water were also sampled at each site $30 \mathrm{~cm}$ below the surface, the depth where M. festivus were fished (Pires et al. 2015), to characterize other water parameters in the laboratory. At the laboratory, dissolved organic carbon (DOC) was quantified and characterized, and the concentration of nutrients $\left(\mathrm{NO}_{2}{ }^{-}, \mathrm{NO}_{3}{ }^{-}\right.$, silicates), free ions $(\mathrm{Ca}, \mathrm{Na}, \mathrm{Cl}, \mathrm{Mg}, \mathrm{K})$ and 12 metals (Al, $\mathrm{V}, \mathrm{Cr}, \mathrm{Mn}, \mathrm{Fe}, \mathrm{Co}, \mathrm{Ni}$, $\mathrm{Cu}, \mathrm{Zn}, \mathrm{As}, \mathrm{Cd}$ and $\mathrm{Pb}$ ) were determined according to the method detailed in Sylvain et al. (2019, 2020). All environmental parameters are the means of three measurements and can be found in the supplementary information section.

Twenty fish specimens were collected at each sampling site using a combination of small seine net-fishing and line fishing (fish collection permit number 29837-18). Shortly after collection, fish were euthanized using a classic MS-222 protocol to dissect a fin clip which was kept in NAP conservation buffer to preserve DNA integrity (Camacho-Sanchez et al. 2013). Samples were frozen at -20 degC right after the dissection and until DNA extraction.

\section{DNA Extraction and Sequencing}

A total of 240 fin samples were collected and processed. Genomic DNA was extracted using the QIAGEN DNeasy(r) Blood and Tissues kit following the manufacturer's instructions. Genomic DNA was then purified with AMPure beads (Beckman Coulter Genomics), according to the manufacturer's instructions. Double strand DNA was quantified using the Qubit(r) 2.0 Fluorometer. GBS libraries and sequencing were done at the IBIS Plateforme d'Analyse Genomique using Pstl and Mspl as restriction enzymes. Barcodes were ligated to the digested DNA fragments and libraries were sequenced using Ion proton technology.

\section{SNPs Calling}

The raw sequence reads were trimmed with Cutadapt (Martin 2011) in order to remove the adapter sequences and sequence quality was assessed using FastQC. The sequences were extracted and trimmed to $80 \mathrm{bp}$ using process_radtags in STACKS V1.5 (Catchen et al. 2013). After trimming with Cutadapt (Martin 2011) and extracting with process_radtags, samples had an average of $2.91(\mathrm{sd}=0.91)$ million reads $(\mathrm{n}=231)$. Nine samples were discarded during the filtration process because of low-quality sequences and/or insufficient coverage. The STACKS programs for de novo SNP discovery were then run with the following filters and parameters. Firstly, we ran Ustacks considering a population size of " $\mathrm{p}=1$ ", a minimum stack depth of " $\mathrm{m}$ $=3$ ", a distance allowed between stacks of " $\mathrm{M}=3$ ", a maximum number of mismatches allowed of " $\mathrm{N}=$ 5 " and activating the "disable-gapped", "H" and "deleverage" options. We have tested multiple parameter combinations and these parameters lead to an optimal discovery of SNPs, are robust and lead to a low false discovery rate for de novo SNPs discovery (Paris et al. 2017). Afterward, we allowed a distance of "n = 1" between catalogue loci in Cstacks and applied the following filtration and transformation steps: Sstacks to match samples against the catalogue, tsv2bam to transpose the data so it is stored by locus, gstacks to call variant sites in each population and ran populations using a " $\mathrm{p}=4$ ", a " $\mathrm{r}=0.6$ " and the option "vcf" to produce a variant call format (VCF) file (Rochette and Catchen 2017). These filtration 
steps led to 428,095 putative SNPs across $M$. festivus' genome. SNPs with heterozygosity rates higher than 0.5 were filtered out, which is considered stringent. The resulting VCF file was further filtered using the script "05_filter_vcf_fast.py" from stacks workflow (https://github.com/enormandeau/stacks_workflow) with the following parameters: a minimum allele coverage to keep genotypes of " $\mathrm{m}=3$ ", a minimum percent of genotype data per population of "percent_genotype $=60$ ", a maximum number of populations that can fail percent genotypes of "max_pop_fail $=0$ " and a minimum number of samples with rare alleles of "min_mas $=2$ ". In total, 41,268 SNPs were conserved after these filtration steps, keeping only non-duplicated loci and removing SNPs in linkage. Overall missing data for the dataset was $11.70 \%$. Also, we screened for high relatedness between samples and estimated the mean heterozygosity rate at each site using the function "genetic_diff" from the library vcfR in R (Knaus and Grunwald 2017).

\section{Data analysis}

\section{Mesonauta festivus Phylogeographic History}

Based on our 41,268 SNPs dataset ( $\mathrm{n}=231$ ), we used ADMIXTURE, a program that estimates individual ancestry from SNP datasets (Alexander and Lange 2011), to calculate the posterior membership probability of each sample considering two to eight potential genetic clusters in the dataset. Then, we selected the number of biologically significant genetic clusters present in the data based on a combination of crossvalidation error values from ADMIXTURE, the goodness of fit (BIC) measures from the "find.cluster" function from Adegenet (Jombart 2008) in R and the analysis of multiple membership probability plots from ADMIXTURE. In addition, pairwise fixation indexes (Fst) were estimated assuming four genetic clusters with the "stamppFst" function from StAMPP (Pembleton et al. 2013) in R. We produced a heatmap to illustrate the linearized Fst/(1-Fst), as they are more adapted to detect scenarios of isolation by distance in linear landscapes like riverscapes (Rousset 1997).

We conducted a multiple linear regression on distance matrices (MRM) (Lichstein 2007), an extension of the partial Mantel analysis. We considered a pairwise "Fst/(1-Fst)" matrix as the dependent variable and used three explanatory matrices: (1) a matrix with pairwise river course distances between sites for isolation by distance; (2) a matrix indicating whether pairs of sites are from the same water type ( same $=0$, different $=$ 1) for isolation by ecology and (3) a matrix indicating whether pairs of sites are connected by downstream water flow (flow-connected $=0$, flow-unconnected $=1$ ) for isolation by unidirectional downstream water currents. The linear MRM was run with 1000 permutations to assess the significance and the power of the three explanatory variables at explaining the genetic dissimilarity between the sampled sites. To visualize the results from the MRM analysis, we produced three simple Mantel tests (Mantel 1967) using the function "mantel.randtest" from the package ade4 in $\mathrm{R}$ to look for one by one linkage between the pairwise linearized Fst/(1-Fst) matrix and the three explanatory matrices used in the MRM.

\section{Environmental association study}

Water Type Comparison Using Environmental Parameters

At each site, water type was assessed based on visual observations and literature research prior to the environmental parameters' characterization. Using the library factoextra in $R$, we calculated a principal component analysis (PCA) on normalized environmental parameters, normalized as deviations from the mean, between sites. It was used to assess which environmental variables were associated with certain water types. The EAS required environmental variables not to be strongly correlated with each other. For this reason, we selected 5 environmental parameters not strongly associated with each others which can differentiate black and white water sites in our study. The selected environmental parameters were the concentration of silicate in suspension $(\mu \mathrm{mol} / \mathrm{L})$, concentration of dissolved organic carbonate (DOC) $(\mathrm{mg} / \mathrm{L})$, conductivity $(\mu \mathrm{S} / \mathrm{L})$, productivity $(\mu \mathrm{g}$ of Chla/L) and aluminum concentration $(\mu \mathrm{g} / \mathrm{L})$. Again, using the library factoextra, we produced a biplot to visualize the importance of these environmental parameters in the differentiation of the black and white water types. 


\section{Detecting Genetic-Environment Associations}

Since EAS models do not accept missing data in their genotype files, we used the ADMIXTURE most probable posterior membership probability result of each sample to impute the missing genotypes according to the procedure described on GitHub: enormandeau/stacks_workflow (Alexander and Lange 2011). We used three proven and recently developed approaches of EAS (de Villemereuil et al. 2014, Rellstab et al. 2015, Capblancq et al. 2018, Forester et al. 2018), which are correcting for neutral genetic variation, to detect associations between our 41,268 SNPs and the diverging environmental parameters of the 5 black and 7 white water sites sampled. For the three EAS methods, we used the imputed SNPs dataset as the dependent variable and used the selected normalized environmental parameters and the water type directly, as a qualitative variable (black water $=1$; white water $=-1$ ), as the explanatory variables to detect associations between the SNPs and the environment.

First, a constrained ordination redundancy analysis (RDA) (Forester et al. 2016; Legendre \& Legendre 2012) was performed to detect covariation between loci and environmental predictors. We performed the RDA considering the genotype matrix as the dependent variable and the environmental parameters as explanatory variables. The covariation and multicollinearity between environmental parameters were verified, with the function "vif.cca" from vegan (Oksanen et al. 2019) in $R$, to ensure that the variance inflation factor was below 5 for each variable. We verified for the significance of each constrained axis and plotted the RDA to look how the samples at each site are clustering according to their respective environmental parameters. Afterward, we selected the SNPs which were very significantly associated to a given constrained axis by selecting outliers SNPs assuming 3.5 standard deviation cut-offs (two-tailed p-value $=0.00046$ ). These putatively under selection SNPs were then associated to the environmental variable they are the most strongly correlated to. We plotted the selected SNPs to visualize their distribution according to the selected constrained axes.

Second, we used Baypass 2.1 (Gautier 2015), a Bayesian hierarchical model based on the BayEnv model (Coop et al. 2010), to detect linear associations between environmental predictors and genetic markers. We performed the core model of Baypass 2.1 with the following parameters (-npop 12, -gfile imputed SNPs dataset, -efile environmental parameters normalized as deviations from the mean, burnin 10000 and -pilotlenght 2500). Using a heat map, we compared the resulting covariance matrix of population allele frequencies to the linearized Fst/(1-Fst) heat map to ensure that the neutral genetic variation was adequately computed by Baypass. Afterward, SNPs strongly associated with environmental predictors (eBPis > 1.5) in the "betail_reg_out" file were selected as putatively under selection for a given environmental variable.

Finally, we used a latent factor mixed model (LFMM2) (Caye et al. 2019), a least-squares estimation approach to detect associations between environmental parameters and genotypes. We performed the LFMM using the function "Ifmm2" from the library LEA (Frichot and Francois 2015, Caye et al. 2019) in R. We ran the function "lfmm2.test" with the genomic control activated and considering a linear model. We corrected for the neutral genetic structure by considering four latent factors based on the four genetic clusters previously detected. We verified the validity of the model using a histogram of p-values for each explanatory variable, aiming for a flat histogram with a peak near zero. Afterward, we adjusted the p-value associated to each SNP using a Bonferroni correction and selected significant SNPs (p-value $<0.05$ ) as putatively under selection.

These three programs have proven to be robust and correct for the neutral genetic structure (Capblancq et al. 2018; de Villemereuil et al. 2014; Forester et al. 2018; Rellstab et al. 2015). Nonetheless, we decided to reduce the false positive rate by selecting only loci detected by at least two of the three methods using the function "calculate_overlap" from the package VennDiagram (Chen and Boutros 2011) in R (de Villemereuil et al. 2014). Selected markers associated with environmental variables were then extracted from the full-genotype dataset and visualized using a PCA generated with Vegan (Oksanen et al. 2019) in R. 


\section{Results}

\section{Phylogeographic analysis}

Admixture results considering different number of clusters (2-4) provide compelling information about the genetic structure of the 12 sites sampled [Fig. 2]. When using $[K=2]$, the sites from Rio Negro and Rio Solimões are strongly differentiated. Only CAT and JAR, two sites located downstream from the Rio Negro, have a weak admixture with Rio Negro sites. When using [K=3], the two sites located upstream of the Rio Negro (BAR and NEG) are differentiated, but still show some admixture with sites downstream (CEM and ANA). Nevertheless, BRA does not share posterior membership probability with BAR and NEG even though these sites are close geographically. When using $[K=4]$, sites located far upstream of the Rio Solimões (SOL and TEF) are differentiated from the rest of the Rio Solimões sites. But PIR, a site even further upstream the Rio Solimões, is not differentiated from other downstream lakes. Each subsequent increase of the $\mathrm{K}$-value up to eight led to the differentiation of a new site, $[\mathrm{K}=5]$ differentiates MAN, $[\mathrm{K}=$ 6] differentiates BRA, $[K=7]$ differentiates PIR and $[K=8]$ differentiates JAR [Fig. S1].

According to the results from the cross-validation error values from ADMIXTURE [Fig. S2], the optimal number of clusters for our genetic data is three. Nevertheless, this cross-validation value 0.20966 is close to the one obtained with four clusters 0.20997. The "find.cluster" function from Adegenet led to a similar result since its goodness of the fit (BIC) values reduced more slowly at the fourth cluster [Fig. S3]. Additionally, the posterior membership probability plots [Fig. S1] stopped forming biologically significant clusters after $[\mathrm{K}=4]$, differentiating only one sampling site at the time when adding more clusters. For this reason, we completed the phylogeographic analysis assuming that our full SNPs dataset is represented by four genetic clusters.

According to the Admixture results [Fig. 2], some sites that are close to each other are showing a strong genetic differentiation with each others. For instance, there is a first genetic gap (i.e., a disproportional genetic distance compared to the river course distance separating sites) at the confluence of the Rio Negro and Rio Solimões. Additionally, there is another second genetic gap between BRA and NEG, and a third one between TEF and PIR. These genetic gaps are detectable in every neutral population structure analysis that we have produced; the Admixture results [Fig. 2] and the high pairwise Fst values [Fig. 3].

In the first genetic gap, white water sites are always more closely related to other white water sites, and vice versa [Fig. 2]. However, the inverse is observed in the second genetic gap, where BRA (white water) has a higher relatedness with ANA (black water) than NEG (black water) has [Fig 2 and 3]. This migration pattern, migrating downstream preferentially from white water to black water, is inverse to the one observed at the Rio Negro-Solimões confluence (The first genetic gap). The third genetic gap is at the confluence of the Rio Tefé and Solimões. Effectively, the genetic distance between SOL and PIR is disproportionately big when compared to the small geographic distance separating the sites. Additionally, SOL (white water) is more closely related to TEF (black water) than PIR (white water). This result is detectable in the Admixture results [Fig. 2], where PIR shares a common posterior membership with other downstream Rio Solimões sites, and TEF and SOL are clustered apart. However, according to the pairwise linearized Fst/(1-Fst) heatmap, TEF and SOL are not much more genetically distant to other downstream Rio Solimões sites than PIR [Fig. $3]$.

The multiple regression on distance matrices $(\mathrm{MRM})$ detected a significant association between the pairwise linearized Fst/(1-Fst) matrix and both the river course distance (p-value $=0.021$ ) and the connectivity $(\mathrm{p}$-value $=0.001)$ matrices. The relation between the genetic distance and the water type similarity matrix was not significant $(\mathrm{p}$-value $=0.571)$. When using both the river course distance and the downstream connectivity matrices, $59.23 \%$ of the dependent matrix is explained by the linear model produced. According to the one-by-one Mantel tests [Fig. S4], the pairwise genetic distances between sites are moderately correlated with the pairwise river course distances (correlation coefficient of 0.54 with a p-value of 0.004$)$. In the same way, there is a strong correlation between the pairwise genetic distances 
and downstream water flow connectivity (correlation coefficient of 0.71 with a p-value of 0.001 ) and a nonsignificant correlation between genetic distances and the water type similarity matrix (correlation coefficient of 0.25 with a p-value $>0.05)$.

\section{Environmental Association Study}

As seen in the physicochemical parameters biplot using the five selected environmental parameters [Fig. 4], differences in water physicochemical characteristics can differentiate the two water types. Black water sites were characterized by higher $\mathrm{DOC}$ and $\mathrm{Al}$ concentrations and lower $\mathrm{pH}$, while white water sites had higher amounts of silicate in suspension, as well as higher conductivity and Chl a concentration [Table 1 and Fig. S5].

All six axes of the RDA were significant ( $\mathrm{p}$-value $<0.05$ ) and used for the detection of associations between the genotypes and environmental predictors. The corrected sum of the variance explained by the environmental predictors in the redundancy analysis is $4.93 \%$. Sample representation in the RDA according to the explanatory variables was unrelated to their respective genetic clusters [Fig. S6]. A total of 584 unique SNPs were associated to the environmental predictors in the RDA. From these, 45 were associated to aluminum concentration, 29 to productivity, 74 to conductivity, 44 to DOC concentration, 357 to silicate concentration and 35 directly to water types [Fig. S7]. For Baypass2, the neutral genetic structure estimated by the program [Fig. S8] is concordant with the Fst heatmap previously produced [Fig. 3]. A total of 307 unique SNPs had an eBPis superior to 1.5 and were considered as putatively under selection. From these, 178 were associated to aluminum concentration, 63 to productivity, 60 to conductivity, 5 to DOC concentration, 21 to silicate concentration and 15 directly to water type. In 35 occurrences, SNPs were associated to two environmental variables. For the LFMM2, a total of 367 unique SNPs had a significant p-value after the Bonferroni correction. From these, 13 were associated to aluminum concentration, 215 to productivity, 107 to conductivity, 4 to DOC concentration, 117 to silicate concentration and 24 directly to water type [Fig. S9]. In 113 occurrences, SNPs were associated to multiple environmental variables. From these SNPs, 172 were found in at least 2 methods and kept for the following analyses [Fig. 5].

Yet, the 172 selected SNPs resulting from our EAS are not structuring the samples according to their water type. According to the PCA using the water type associated SNPs [Fig. 6], samples are clustering according to their watershed of origin [Fig. 6B] and not according to their water type [Fig. 6C]. Samples from the two main Amazonian watersheds are well differentiated by PC1, which explains $26.56 \%$ of the variation in the genetic matrix. Additionally, BRA (white water) is clustering with black water sites from the Rio Negro (i.e., ANA, CEM, NEG and BAR). In contrast, TEF and SOL (respectively black and white water sites) seemed to be isolated from the other Solimões River sites, which is concordant with our previous results [Fig. 2 and 3]. When compared to a PCA using the full 41,268 SNPs [Fig. S10], the general clusters stay the same. The only major difference is in the clustering of SOL and TEF with the other sites from the Solimões watershed and the higher dispersion of the sites from Rio Negro along PC2. Again, the differences in water type between sites do not seem to be the main structuring factors in the data.

\section{Discussion}

Using a genotyping by sequencing approach, we provide a unique and robust dataset supporting the structuration of $M$. festivus populations by evolution through vicariance events. Our holistic sampling design, combining 12 sites from a wide territory, 231 M. festivus samples and an optimized SNPs calling bioinformatic pipeline render remarkably different conclusions than what other similar studies obtained (Cooke et al. 2009; 2014; 2012a; b; Beheregaray et al. 2015). Using a wider sampling design and a combination of phylogeographic and environmental association models, we challenge the previous ecological speciation hypothesis (i.e., genetic structure is linked to diverging water types) and show strong evidence that water type has a low-structuring power on $M$. festivus populations. Our results support a much more important influence of 
vicariance events associated with the Amazon's formation and isolation by unidirectional downstream water current on structuring populations in this clade.

\section{Phylogeographic analysis}

According to the Admixture posterior membership probability plots [Fig. S1], the cross-validation error values from ADMIXTURE [Fig. S2] and the BIC values from "find.cluster" [Fig. S3], four genetic populations were detected across our 12 sampling sites. The geographic structuring of these four genetic populations can be used to infer the phylogeographic history of $M$. festivus, its colonization potential and to infer on the evolutionary history of closely related clades.

The first genetic group detected is formed by five Rio Solimões sites: CAT, JAR, JAC, MAN and PIR. Despite the small river course distance separating them, it appears that an important genetic distance separates CAT, JAR, JAC and MAN from the sites sampled in the Rio Negro [Fig. 2]. This first genetic gap [Fig. 2], between the Rio Negro and Solimões, has already been assessed in a series of scientific papers based on similar sampling designs (Cooke et al. 2009; 2012a; b; 2014; Beheregaray et al. 2015). In these studies, this genetic gap was associated with ecological speciation, an evolutionary process caused by the presence of an ecotone like the black and white water confluence. Strikingly, fish sampled at CAT are more closely related to PIR than to CEM, located respectively at 631 and $71 \mathrm{~km}$ from CAT [Fig. 1]. Here, the genetic distances seem to be unrelated to the geographic distance, but strongly correlated to other variables such as water type differences and isolation by unidirectional downstream currents. While this result is in concordance with the ecological speciation hypothesis and the results from Cooke et al. (2009; 2014; 2012a; b), it ultimately does not lead us to the same evolutionary conclusions.

Indeed, we observed a second genetic gap at the confluence of the Negro and Branco Rivers [Fig. 2], forming our second genetic group composed of the sites BAR and NEG. This genetic divergence has not been recorded in previous Amazonian population genetic studies, demonstrating the advantage of using a larger sampling design. While it might be interpreted as evidence of the divergence between the black (NEG) and white (BRA) water types, this conjecture is refuted by the higher relatedness between BRA and ANA, respectively white and black water sites, than between NEG and ANA, both black water, in the Admixture results and the Pairwise linearized Fst heatmap [Fig. 2-3]. This is despite the drastic environmental shift separating ANA and BRA, and both sites being at a similar river course distance from ANA, respectively 215 and $225 \mathrm{~km}$ for NEG and BRA. To reiterate, this result supports that gene flow is stronger between sites with different water types and that water type is not an important migration barrier for $M$. festivus at these sites. These genetic patterns are similar when comparing BRA and NEG with CEM [Fig. 2 and 3]. These sites: BRA, ANA and CEM, are forming the third genetic population observed.

Here, the strong genetic divergence between NEG and BRA could be caused by the strong unidirectional downstream water current that prevents the migration of $M$. festivus from the Rio Branco to upstream of the Rio Negro and vice versa. This downstream biased gene flow could lead to a partial isolation of upstream $M$. festivus populations, which admix in downstream rivers. Ultimately, this should lead to a downstream increase in intraspecific genetic diversity at the confluence of large rivers where multiple populations that are upstream and isolated by current genetic populations meet (Paz-Vinas et al. 2015). Systematically, the sites located downstream of the main rivers (i.e., CEM, ANA, CAT and JAR) did have a higher mean heterozygosity rate than their upstream relatives (i.e., NEG, BRA and MAN) [Table 1]. In fact, the sites with the lowest heterozygosity were the ones located the most upstream (i.e., BRA, BAR, PIR and TEF). Similarly, freshwater fish diversity hotspots in the Amazon watershed are usually located at the crossing of large rivers near the meeting of large watercourses (Oberdorff et al. 2019). For instance, the highest total species richness is found at the confluence of the Negro and Solimões Rivers and other large river confluences show similar diversity patterns, for example the Tapajós-Amazon Rivers confluence and BrancoNegro Rivers (Oberdorff et al. 2019). This is supporting the hypothesis of evolution by allopatry, where neutral evolutionary processes in fish populations located in isolated rivers were followed by a reconnection of the waterways and a mix of the newly formed and reproductively isolated species. 
The fourth and last genetic population is formed by two sites located in the upper Rio Solimões, near the Rio Téfé: SOL and TEF. The third genetic gap is located between SOL and PIR, a site located only $6 \mathrm{~km}$ upstream of SOL [Fig. 2]. According to the linearized Fst/(1-Fst) heatmap, this genetic population does not arbour an important genetic dissimilarity with the other Rio Solimões sites [Fig. 3]. Yet, the pairwise Fst Values between SOL and PIR are similar to Fst values with sites located more than $500 \mathrm{~km}$ downstream the Rio Solimões. Therefore, TEF (black water) seems to lead to higher gene flow into SOL (white water) than PIR (white water) does. While this result goes against the ecological speciation hypothesis, the dissimilarity between SOL and PIR could, again, be caused by isolation by strong downstream water currents. We selected the site SOL as a site located right between PIR and TEF, two sites characterized by drastically divergent environmental conditions [Table 1]. It is possible that the river architecture between TEF and SOL is more favourable to gene flow coming from TEF than from PIR for M. festivus. Either way, this again supports that water type is not an important migration barrier for $M$. festivus since the populations from different water types are more genetically alike to each other's than the site from the same water type.

According to the MRM analysis results, most of the gene flow between the sampled sites happened between sites connected by downstream water flow, with higher amounts of gene flow happening between sites located close to each other, irrespectively of the water type at each site [Fig. S4]. In this sense, the water type at each site had a non-significant and low power at explaining the genetic distance between sites. On the contrary, the connectivity between sites was very significant and inversely correlated to the genetic distance between sites. In the same way, sites separated by a small pairwise river course distance were more genetically related. According to these results, the divergent water types do not significantly affect the migration rate between sites for M. festivus. Conversely, the downstream water flows connectivity and the distance between rivers seemed to play a much more important role at structuring $M$. festivus genetic populations.

According to our results based on neutral loci, the three genetic gaps detected are caused by a combination of isolation by strong unidirectional water current and past evolution by allopatry between watersheds that recently reconnected. While the diverging environmental conditions must certainly have at least some effects on fish evolution, due to differences in productivity, food availability, species assemblages, environmental pressures, physiological demands, etc., we argue that isolation by strong downstream water current and past isolation by geological processes played a much more important role in shaping the genetic structure of $M$. festivus populations.

\section{Environmental Association Study}

While we did not detect an important impact of the water types on the structure of $M$. festivus genetic populations using neutral loci, the effect of an ecologically driven change in specific allelic frequencies in presence of gene flow could still be detected using genotype to environment association models. We conducted a complete environment to genotype association study (EAS) aiming to identify SNPs strongly associated with selected environmental variables and directly to water type. We chose to use DOC concentration, chlorophyll a concentration, conductivity, silicate in suspension and dissolved aluminum concentration since other authors have previously employed these to characterize black and white waters (Junk et al. 2011, 2015). While a low $\mathrm{pH}$ has proven to be a major characteristic of the black water environment (Ríos-Villamizar et al. 2013), its strong covariation with other parameters required its exclusion from the analyses. The aforementioned parameters have a very good power at differentiating black and white water sites in our study [Fig. 4]. If water type is responsible for a strong ecological speciation in our system, we expect to detect a strong pattern of differentiation between sites of different water types at the 172 SNPs associated with water type and its associated physicochemical parameters [Fig. 5]. Also, samples should not cluster according to their watershed of origin.

In a PCA, the clustering of samples according to the SNPs associated to the environment [Fig. 6] is similar to the result obtained using the full dataset [Fig. S10], which is compatible with the scenario of neutral divergence in allopatry. Likewise, the dominant influence of neutral evolutionary forces, mutation and genetic drift in conditions of low gene flow between certain populations, contrasts with the low influence of 
directional evolutionary forces in the genetic structure of environmentally associated SNPs. When clustered by watershed, samples are very well differentiated in the PCA plot [Fig. 6B], while clustering the samples by their water type gives a much more admixed PCA plot [Fig. 6C]. This is strong evidence that the presence of divergent water types is not one of the main evolutionary factors and that neutral evolutionary processes have a much stronger impact on the differentiation of these populations. This is even though we corrected for the neutral genetic structure in the three EAS methods. Since we only sampled a fraction of $M$. festivus' genome and did not have access to a reference genome to map for SNPs associated with genes of interest, this result does not rule out the possibility that other genes could be positively selected in a specific water type. Doing an analysis with a reference genome could lead to the discovery of key genes that affect the fitness of $M$. festivus individuals in each environment. When combined with our previous analyses, the EAS results provide very strong evidence that evolution by ecological speciation did not have an important influence on M. festivus population structure in Amazonia.

\section{Refuting Previous Assumptions of Strong Ecological Speciation}

The recently accepted assumption that ecological speciation was a major Teleostean evolution driver in the tropics seems to have been derived from observations of a site effect in papers with similar experimental designs (Cooke et al. 2009; 2012; 2012a; 2015). Our results support that, in these papers, the structuring effect caused by the water type was potentially confounded with the effect of a strong unidirectional water flow at the sampled sites. Furthermore, adding new sites to these past studies could lead them to different conclusions. For instance, if we consider only the sites that were used by Cooke et al. $(2009 ; 2012 ; 2012 a$; 2015) in our results, it leads us to very similar results and conclusions, and this is even though this series of previous studies focused on different species that are genetically distant from $M$. festivus. For these papers, Cooke et al. sampled the genetic gap happening at the confluence of the Rio Negro and Solimões. Our genetic data behaves identically to theirs at these sites and we detected an extensive genetic distance between the Rio Negro and Solimões populations. It is only after adding the data from sampling sites at two other black and white water confluences that we detected evidence that adaptative divergence to specific water types was not the main driver of population structure in $M$. festivus. There seems to be a real possibility that adding new sites to Cooke's studies would lead to results like ours. The application of a wide sampling design and a deep sequencing approach have previously resolved fine-scale phylogeographic patterns in other teleostean species (Fairweather et al. 2018; Fang et al. 2018).

\section{A Demographic Scenario Based on the Amazon's Geological History}

Our study detected three main genetic gaps at the confluences of: (1) the Negro and Solimões Rivers, (2) Branco and Negro Rivers, and (3) Lago Tefé and Solimões Rivers. What do these three genetic gaps have in common? First, they all represent a confluence of black and white water rivers. Second, gene flow is always going downstream, which could mean the water current is the primary driver of gene flow for M. festivus. Considering its physiology and sedentary behaviour (Pires et al. 2015), strong current velocity represents an important environmental gene flow barrier for M. festivus. Additionally, there have been anecdotes of fishers seeing M. festivus floating in high current while imitating a dead leaf (Pires et al. 2015), which could explain why there are sources of downstream gene flow over very long distances. Lastly, the three confluences result from the formation of the modern Amazon during the late Pliocene, between 2.5 Ma and $700 \mathrm{Ka}$ (Campbell et al. 2006; Ribas et al. 2012).

The formation of the modern Amazon is relatively recent and has shown to be one of the main causes of the Amazonian Animalia terrestrial diversity boom (Albert et al. 2018; Araújo-Silva et al. 2017; Lynch Alfaro et al. 2015). Numerous authors support the Pleistocene refugia hypothesis, assuming that Amazonia diversity is partly due to geographic isolation caused by geologic processes and salt-water incursion events (Farias and Hrbek 2008, Braganca and Costa 2018, da Rocha and Kaefer 2019). Amazonian cichlids are much older, dating from the separation from their African sister clade, and have evolved mostly in riverine ecosystems (Concheiro Perez et al. 2007). For this reason, M. festivus, a ubiquitous Cichlid species, is a 
good model to understand the impact of the formation of the modern Amazon on fish evolution. The modern transcontinental Amazon got established 2.5 Mya, when the west and east territories were divided in two (Campbell et al. 2006). The water drainage led to sediment accumulation, creating thousands of small lakes and rivers. The Negro and Branco Rivers were formed approximately between 1.0 to 0.7 Mya and Tefe River approximately between 2.0 to 1.0 Mya (Ribas et al. 2012). M. festivus was present well before the formation of these rivers and probably colonized the new downstream environments as they formed. As previously mentioned, M. festivus has a very good colonization potential of new environments and show strong proof of downstream migration. In this sense, the founding population of $M$. festivus probably came from the Andes, in the west, and admixed in multiple downstream rivers, irrespective of their water type. Likewise, the genetic gaps detected at the confluences of black and white water rivers are probably related to the formation of the rivers (geologic history) rather than the confounding effect of the water type. As a result, the strong genetic divergence between the $M$. festivus populations of the Negro and Solimoes rivers probably stems from geological processes that led to a neutral evolution by allopatry.

M. festivus low upstream migration potential (Pires et al. 2015) must have slowed the admixture process after the reconnection of the waterways as observed in the present watershed structures. Considering this and the similarity between our results and those from Cooke et al. (2009; 2012; 2012a; 2015), multiple Amazonian fish species could have evolved in these same vicariant conditions, potentially leading to the genesis of many new species, who could have dispersed after the connection of the waterways. In order to test whether some population divergence times are linked to important geological events, it would be interesting to do a demographic analysis with a molecular clock estimate based on M. festivus mitochondrial DNA from sites positioned close to past major geologic processes.

Our study aimed to investigate the support for the two main speciation hypotheses (ecological and allopatric) used to explain the evolution of M. festivus within the Amazon basin. We showed strong evidence that the divergent physicochemical characteristics between black and white water have a weak structuring power on M. festivus populations in the Amazonian watershed. Furthermore, our results challenge the recently suggested ecological speciation hypothesis explaining fish diversification in Amazonia. Unlike previous studies focusing on a single confluence between black and white water, our extensive sampling design comprising 12 populations of $M$. festivus detected a genetic structure congruent with isolation by unidirectional downstream water current, past geologic events, and waterways connectivity shift. While the Brazilian Amazon supports one of the richest fish faunas on Earth, our comprehension of the evolutionary processes which shaped its biodiversity is still lacking. Understanding the origin of such richness would help us protect its diversity. Our study not only constitutes a step forward in understanding these important processes but also provides a conceptual framework that should benefit the sampling designs of future investigations on this matter.

\section{Conclusion}

Our study aimed to investigate the support for the two main speciation hypotheses (ecological and allopatric) used to explain the evolution of $M$. festivus within the Amazon basin. We showed strong evidence that the divergent physicochemical characteristics between black and white water have a weak structuring power on M. festivuspopulations in the Amazonian watershed. Furthermore, our results challenge the recently suggested ecological speciation hypothesis explaining fish diversification in Amazonia. Unlike previous studies focusing on a single confluence between black and white water, our extensive sampling design comprising 12 populations of $M$. festivus detected a genetic structure congruent with isolation by unidirectional downstream water current, past geologic events, and waterways connectivity shift. While the Brazilian Amazon supports one of the richest fish faunas on Earth, our comprehension of the evolutionary processes which shaped its biodiversity is still lacking. Understanding the origin of such richness would help us protect its diversity. Our study not only constitutes a step forward in understanding these important processes but also provides a conceptual framework that should benefit the sampling designs of future investigations on this matter. 


\section{ACKNOWLEDGEMENTS}

We gratefully acknowledge help from all members of the Derome and Val laboratory. Thank you to the IBIS Plateforme d'Analyse Genomiquefor the sequencing and to ICMBIO/Instituto Chico Mendes de Conservacao da Biodiversidade for in situ support for fish collection and for issuing the permit to transport biological samples (permit number 29837/18). This research was part of the ADAPTA project at INPA and was supported by the INCT ADAPTA (CNPq/FAPEAM) and INPA/MCTI grants to ALV (ORCID: 0000-0002-3823-3868), the Natural Sciences and Engineering Research Council of Canada (NSERC) Discovery grant (grant \#6333) and the Canada-Brazil Awards Joint Research Project to ND (ORCID: 00000002-2509-6104), and the NSERC BESC M Alexander-Graham-Bell, the FRQNT Master Scholarship grant, the MITACS GlobaLink grant, the Ressources Aquatiques Quebec (RAQ) internship grant and the Andre Darveau grant to NL (ORCID: 0000-0003-1198-9435). Additionally, we would like to thank Martin Laporte, Tommy Pontbriand and Jeremie Boucher-Fontaine for their help reviewing the manuscript.

\section{AUTHOR CONTRIBUTIONS}

F.S., N.L., E.N., N.D. and A.V. designed the experiment. F.S., N.L., A.H., N.D. and A.V. organized sampling expeditions. F.S., N.L., A.H. and N.D. sampled fish during field expeditions. N.L., F.S., A.H. and N.D. processed samples in the laboratory (fish dissections and DNA extractions). N.L. and E.N. performed bioinformatic analyses. N.L. wrote the manuscript. All authors reviewed the manuscript.

\section{ETHICAL APPROVAL}

This study was carried out in accordance with the recommendations of the Ethics Committee for the Use of Animals of the Instituto Nacional de Pesquisas da Amazonia (INPA). The permit (number 29837-18 as of 23 March 2021) was approved by the Ethics Committee for the Use of Animals of INPA.

\section{DATA AVAILABILITY STATEMENT}

The scripts and the datasets used for the statistical analysis of this project are freely available on the Open Science Framework (DOI 10.17605/OSF.IO/2J5FH). Raw sequence reads are deposited in the SRA (BioProject XXX) and metadata are also stored in the SRA (BioProject XXX) using the MIxS package MIGS.eu.5.0.

\section{References}

Abreu, J. M. S., A. C. S. Saraiva, J. S. Albert, and N. M. Piorski. 2020. Paleogeographic influences on freshwater fish distributions in northeastern Brazil. Journal of South American Earth Sciences 102:102692.

Albert, J. S., P. Val, and C. Hoorn. 2018. The changing course of the Amazon River in the Neogene: center stage for Neotropical diversification. Neotropical Ichthyology 16:e180033.

Alexander, D. H., and K. Lange. 2011. Enhancements to the ADMIXTURE algorithm for individual ancestry estimation. BMC Bioinformatics 12:246.

Araujo-Silva, L. E., L. S. Miranda, L. Carneiro, and A. Aleixo. 2017. Phylogeography and diversification of an Amazonian understorey hummingbird: paraphyly and evidence for widespread cryptic speciation in the Plio-Pleistocene. Ibis 159:778-791. 
Beheregaray, L., G. Cooke, N. Chao, and E. Landguth. 2015. Ecological speciation in the tropics: insights from comparative genetic studies in Amazonia. FRONTIERS IN GENETICS 5.

Berger, A., and M. F. Loutre. 2010. Modeling the 100-kyr glacial-interglacial cycles. Global and Planetary Change 72:275-281.

Braganca, P. H. N., and W. J. E. M. Costa. 2018. Time-calibrated molecular phylogeny reveals a MiocenePliocene diversification in the Amazon miniature killifish genus Fluviphylax (Cyprinodontiformes: Cyprinodontoidei). Organisms Diversity \& Evolution 18:345-353.

Camacho-Sanchez, M., P. Burraco, I. Gomez-Mestre, and J. A. Leonard. 2013. Preservation of RNA and DNA from mammal samples under field conditions. Molecular Ecology Resources 13:663-673.

Campbell, K. E., C. D. Frailey, and L. Romero-Pittman. 2006. The Pan-Amazonian Ucayali Peneplain, late Neogene sedimentation in Amazonia, and the birth of the modern Amazon River system. Palaeogeography Palaeoclimatology Palaeoecology 239:166-219.

Capblancq, T., K. Luu, M. G. B. Blum, and E. Bazin. 2018. Evaluation of redundancy analysis to identify signatures of local adaptation. Molecular Ecology Resources 18:1223-1233.

Catchen, J., P. A. Hohenlohe, S. Bassham, A. Amores, and W. A. Cresko. 2013. Stacks: an analysis tool set for population genomics. Molecular Ecology 22:3124-3140.

Caye, K., B. Jumentier, J. Lepeule, and O. Francois. 2019. LFMM 2: Fast and Accurate Inference of Gene-Environment Associations in Genome-Wide Studies. Molecular Biology and Evolution 36:852-860.

Chen, H., and P. C. Boutros. 2011. VennDiagram: a package for the generation of highly-customizable Venn and Euler diagrams in R. BMC bioinformatics 12:35.

Concheiro Perez, G. A., O. Říčan, G. Ortí, E. Bermingham, I. Doadrio, and R. Zardoya. 2007. Phylogeny and biogeography of 91 species of heroine cichlids (Teleostei: Cichlidae) based on sequences of the cytochrome $\mathrm{b}$ gene. Molecular Phylogenetics and Evolution 43:91-110.

Cooke, G. M., N. L. Chao, and L. B. Beheregaray. 2009. Phylogeography of a flooded forest specialist fish from central Amazonia based on intron DNA: the cardinal tetra Paracheirodon axelrodi . Freshwater Biology 54:1216-1232.

Cooke, G. M., N. L. Chao, and L. B. Beheregaray. 2012a. Marine incursions, cryptic species and ecological diversification in Amazonia: the biogeographic history of the croaker genus Plagioscion(Sciaenidae). Journal of Biogeography 39:724-738.

Cooke, G. M., N. L. Chao, and L. B. Beheregaray. 2012b. Divergent natural selection with gene flow along major environmental gradients in Amazonia: insights from genome scans, population genetics and phylogeography of the characin fish Triportheus albus . Molecular Ecology 21:2410-2427.

Cooke, G. M., N. L. Chao, and L. B. Beheregaray. 2012c. Natural selection in the water: freshwater invasion and adaptation by water colour in the Amazonian pufferfish. Journal of Evolutionary Biology 25:1305-1320.

Cooke, G. M., E. L. Landguth, and L. B. Beheregaray. 2014. Riverscape Genetics Identifies Replicated Ecological Divergence Across an Amazonian Ecotone. Evolution 68:1947-1960.

Coop, G., D. Witonsky, A. Di Rienzo, and J. K. Pritchard. 2010. Using Environmental Correlations to Identify Loci Underlying Local Adaptation. Genetics 185:1411-1423.

Fairweather, R., I. R. Bradbury, S. J. Helyar, M. de Bruyn, N. O. Therkildsen, P. Bentzen, J. HemmerHansen, and G. R. Carvalho. 2018. Range-wide genomic data synthesis reveals transatlantic vicariance and secondary contact in Atlantic cod. Ecology and Evolution 8:12140-12152.

Fang, B., J. Merila, F. Ribeiro, C. M. Alexandre, and P. Momigliano. 2018. Worldwide phylogeny of three-spined sticklebacks. Molecular Phylogenetics and Evolution 127:613-625. 
Farias, I. P., and T. Hrbek. 2008. Patterns of diversification in the discus fishes (Symphysodon spp . Cichlidae) of the Amazon basin. Molecular Phylogenetics and Evolution 49:32-43.

Filoso, S., and M. R. Williams. 2000. The hydrochemical influence of the Branco River on the Negro River and Anavilhanas archipelago, Amazonas, Brazil. Archiv fur Hydrobiologie:563-585.

Forester, B. R., M. R. Jones, S. Joost, E. L. Landguth, and J. R. Lasky. 2016. Detecting spatial genetic signatures of local adaptation in heterogeneous landscapes. Molecular Ecology 25:104-120.

Forester, B. R., J. R. Lasky, H. H. Wagner, and D. L. Urban. 2018. Comparing methods for detecting multilocus adaptation with multivariate genotype-environment associations. Molecular Ecology 27:22152233 .

Frichot, E., and O. Francois. 2015. LEA: An R package for landscape and ecological association studies. Methods in Ecology and Evolution 6:925-929.

Gautier, M. 2015. Genome-Wide Scan for Adaptive Divergence and Association with Population-Specific Covariates. bioRxiv:023721.

Grant, G. R., T. R. Naish, G. B. Dunbar, P. Stocchi, M. A. Kominz, P. J. J. Kamp, C. A. Tapia, R. M. McKay, R. H. Levy, and M. O. Patterson. 2019. The amplitude and origin of sea-level variability during the Pliocene epoch. Nature 574:237-241.

Guichoux, E., L. Lagache, S. Wagner, P. Chaumeil, P. Leger, O. Lepais, C. Lepoittevin, T. Malausa, E. Revardel, F. Salin, and R. J. Petit. 2011. Current trends in microsatellite genotyping. Molecular Ecology Resources 11:591-611.

Holland, A., C. M. Wood, D. S. Smith, T. G. Correia, and A. L. Val. 2017. Nickel toxicity to cardinal tetra (Paracheirodon axelrodi) differs seasonally and among the black, white and clear river waters of the Amazon basin. Water Research 123:21-29.

Hoorn, C., F. P. Wesselingh, H. ter Steege, M. A. Bermudez, A. Mora, J. Sevink, I. Sanmartin, A. SanchezMeseguer, C. L. Anderson, J. P. Figueiredo, C. Jaramillo, D. Riff, F. R. Negri, H. Hooghiemstra, J. Lundberg, T. Stadler, T. Sarkinen, and A. Antonelli. 2010. Amazonia Through Time: Andean Uplift, Climate Change, Landscape Evolution, and Biodiversity. Science 330:927-931.

Hubert, N., F. Duponchelle, J. Nunez, C. Garcia-Davila, D. Paugy, and J.-F. Renno. 2007. Phylogeography of the piranha generaSerrasalmus and Pygocentrus : implications for the diversification of the Neotropical ichthyofauna. Molecular Ecology 16:2115-2136.

Hubert, N., and J.-F. Renno. 2006. Historical biogeography of South American freshwater fishes. Journal of Biogeography 33:1414-1436.

Jari Oksanen and F. Guillaume Blanchet and Michael Friendly and Roeland Kindt and Pierre Legendre and Dan McGlinn and Peter R. Minchin and R. B. O'Hara and Gavin L. Simpson and Peter Solymos and M. Henry H. Stevens and Eduard Szoecs and Helene Wagner. 2019. vegan: Community Ecology Package.

Jombart, T. 2008. adegenet: a R package for the multivariate analysis of genetic markers. BIOINFORMATICS 24:1403-1405.

Junk, W. J., M. T. F. Piedade, J. Schongart, M. Cohn-Haft, J. M. Adeney, and F. Wittmann. 2011. A Classification of Major Naturally-Occurring Amazonian Lowland Wetlands. Wetlands 31:623-640.

Junk, W. J., F. Wittmann, J. Schoengart, and M. T. F. Piedade. 2015. A classification of the major habitats of Amazonian black-water river floodplains and a comparison with their white-water counterparts. Wetlands Ecology and Management 23:677-693.

Knaus, B. J., and N. J. Grunwald. 2017. vcfr: a package to manipulate and visualize variant call format data in R. Molecular Ecology Resources 17:44-53. 
Latrubesse, E. M., and E. Franzinelli. 2005. The late Quaternary evolution of the Negro River, Amazon, Brazil: Implications for island and floodplain formation in large anabranching tropical systems. Geomorphology 70:372-397.

Legendre, P., and L. Legendre. 2012. Numerical Ecology. Page (P. Legendre and L. Legendre, Eds.). Elsevier.

Li, Y.-H., and H.-P. Wang. 2017. Advances of genotyping-by-sequencing in fisheries and aquaculture. Reviews in Fish Biology and Fisheries 27:535-559.

Lichstein, J. W. 2007. Multiple regression on distance matrices: a multivariate spatial analysis tool. Plant Ecology 188:117-131.

Lovejoy, N. R., J. S. Albert, and W. G. R. Crampton. 2006. Miocene marine incursions and marine/freshwater transitions: Evidence from Neotropical fishes. Journal of South American Earth Sciences $21: 5-13$.

Lynch Alfaro, J. W., J. P. Boubli, F. P. Paim, C. C. Ribas, M. N. F. da Silva, M. R. Messias, F. Rohe, M. P. Merces, J. S. Silva Junior, C. R. Silva, G. M. Pinho, G. Koshkarian, M. T. T. Nguyen, M. L. Harada, R. M. Rabelo, H. L. Queiroz, M. E. Alfaro, and I. P. Farias. 2015. Biogeography of squirrel monkeys (genus Saimiri ): South-central Amazon origin and rapid pan-Amazonian diversification of a lowland primate. Molecular Phylogenetics and Evolution 82:436-454.

Mantel, N. 1967. The Detection of Disease Clustering and a Generalized Regression Approach. Cancer Research 27:209-220.

Martin, M. 2011. Cutadapt removes adapter sequences from high-throughput sequencing reads. EMBnet.journal 17:10-12.

Moreira, L. S., P. Moreira-Turcq, R. C. Cordeiro, B. Turcq, K. C. Aniceto, M. Moreira-Ramirez, A. P. S. Cruz, S. Caquineau, and V. C. Silva. 2020. Climate and hydrologic controls on late Holocene sediment supply to an Amazon floodplain lake. Journal of Paleolimnology 64:389-403.

Nielsen, R., J. S. Paul, A. Albrechtsen, and Y. S. Song. 2011. Genotype and SNP calling from next-generation sequencing data. Nature Reviews Genetics 12:443-451.

Nores, M. 2004. The Implications of Tertiary and Quaternary Sea Level Rise Events for Avian Distribution Patterns in the Lowlands of Northern South America. Global Ecology and Biogeography 13:149-161.

Oberdorff, T., M. S. Dias, C. Jezequel, J. S. Albert, C. C. Arantes, R. Bigorne, F. M. Carvajal-Valleros, A. De Wever, R. G. Frederico, M. Hidalgo, B. Hugueny, F. Leprieur, M. Maldonado, J. Maldonado-Ocampo, K. Martens, H. Ortega, J. Sarmiento, P. A. Tedesco, G. Torrente-Vilara, K. O. Winemiller, and J. Zuanon. 2019. Unexpected fish diversity gradients in the Amazon basin. Science Advances 5.

Paris, J. R., J. R. Stevens, and J. M. Catchen. 2017. Lost in parameter space: a road map for stacks. Methods in Ecology and Evolution 8:1360-1373.

Paz-Vinas, I., G. Loot, V. M. Stevens, and S. Blanchet. 2015. Evolutionary processes driving spatial patterns of intraspecific genetic diversity in river ecosystems. Molecular Ecology 24:4586-4604.

Pembleton, L. W., N. O. I. Cogan, and J. W. Forster. 2013. StAMPP: an R package for calculation of genetic differentiation and structure of mixed-ploidy level populations. Molecular Ecology Resources 13:946-952.

Pires, T., D. Campos, C. Ropke, J. Sodre, S. Amadio, and J. Zuanon. 2015. Ecology and life-history of Mesonauta festivus : biological traits of a broad ranged and abundant Neotropical cichlid. Environmental Biology of Fishes 98:789-799.

Pires, T. H. S., E. A. Borghezan, V. N. Machado, D. L. Powell, C. P. Ropke, C. Oliveira, J. Zuanon, and I. P. Farias. 2018. Testing Wallace's intuition: water type, reproductive isolation and divergence in an 
Amazonian fish. Journal of Evolutionary Biology 31:882-892.

Potter, P. E. 1997. The Mesozoic and Cenozoic paleodrainage of South America: a natural history. Journal of South American Earth Sciences 10:331-344.

Pritchard, J. K., M. Stephens, and P. Donnelly. 2000. Inference of population structure using multilocus genotype data. Genetics 155:945.

Reis, R. E. 2013. Conserving the freshwater fishes of South America: Conserving South American Freshwater Fishes. International Zoo Yearbook 47:65-70.

Rellstab, C., F. Gugerli, A. J. Eckert, A. M. Hancock, and R. Holderegger. 2015. A practical guide to environmental association analysis in landscape genomics. Molecular Ecology 24:4348-4370.

Ribas, C. C., A. Aleixo, A. C. R. Nogueira, C. Y. Miyaki, and J. Cracraft. 2012. A palaeobiogeographic model for biotic diversification within Amazonia over the past three million years. Proceedings of the Royal Society B: Biological Sciences 279:681-689.

Rios-Villamizar, E. A., M. T. F. Piedade, J. G. Da Costa, J. M. Adeney, and W. J. Junk. 2013. Chemistry of different Amazonian water types for river classification: a preliminary review. Pages 17-28. New Forest, UK.

da Rocha, D. G., and I. L. Kaefer. 2019. What has become of the refugia hypothesis to explain biological diversity in Amazonia? Ecology and Evolution 9:4302-4309.

Rousset, F. 1997. Genetic Differentiation and Estimation of Gene Flow from F-Statistics Under Isolation by Distance. Genetics 145:1219-1228.

Roxo, F. F., C. H. Zawadzki, M. A. Alexandrou, G. J. Costa Silva, M. C. Chiachio, F. Foresti, and C. Oliveira. 2012. Evolutionary and biogeographic history of the subfamily Neoplecostominae (Siluriformes: Loricariidae). Ecology and Evolution 2:2438-2449.

Rundle, H. D., and P. Nosil. 2005. Ecological speciation. Ecology Letters 8:336-352.

Schluter, D. 2001. Ecology and the origin of species. Trends in Ecology \& Evolution 16:372-380.

Schluter, D. 2009. Evidence for Ecological Speciation and Its Alternative. Science 323:737-741.

Sioli, H. 1984. Water chemistry of the Amazon basin: The distribution of chemical elements among freshwaters. Pages 167-199 in H. Sioli, editor. The Amazon: Limnology and landscape ecology of a mighty tropical river and its basin. Springer Netherlands, Dordrecht.

Smart, P. 2017. Redundant publication and salami slicing: the significance of splitting data. Developmental Medicine \& Child Neurology 59:775-775.

Sylvain, F.-E., A. Holland, E. Audet-Gilbert, A. L. Val, and N. Derome. 2019. Amazon fish bacterial communities show structural convergence along widespread hydrochemical gradients. Molecular Ecology $28: 3612-3626$.

Sylvain, F.-E., A. Holland, S. Bouslama, E. Audet-Gilbert, C. Lavoie, A. L. Val, and N. Derome. 2020. Fish Skin and Gut Microbiomes Show Contrasting Signatures of Host Species and Habitat. Applied and Environmental Microbiology 86:e00789-20.

Thom, G., A. T. Xue, A. O. Sawakuchi, C. C. Ribas, M. J. Hickerson, A. Aleixo, and C. Miyaki. 2020. Quaternary climate changes as speciation drivers in the Amazon floodplains. Science Advances 6:eaax4718.

Val, A. L., and V. M. F. Almeida-Val. 1995. Fishes of the Amazon and Their Environment. Springer Berlin Heidelberg. 
Venere, P. C., I. L. Souza, L. K. S. Silva, M. B. D. Anjos, R. R. D. Oliveira, and P. M. Galetti. 2008. Recent chromosome diversification in the evolutionary radiation of the freshwater fish family Curimatidae (Characiformes). Journal of Fish Biology 72:1976-1989.

Venticinque, E., B. Forsberg, R. Barthem, P. Petry, L. Hess, A. Mercado, C. Canas, M. Montoya, C. Durigan, and M. Goulding. 2016. An explicit GIS-based river basin framework for aquatic ecosystem conservation in the Amazon. Earth System Science Data 8:651-661.

de Villemereuil, P., E. Frichot, E. Bazin, O. Francois, and O. E. Gaggiotti. 2014. Genome scan methods against more complex models: when and how much should we trust them? Molecular Ecology 23:2006-2019.

Yamaguchi, R., and Y. Iwasa. 2013. First passage time to allopatric speciation. Interface Focus 3:20130026.

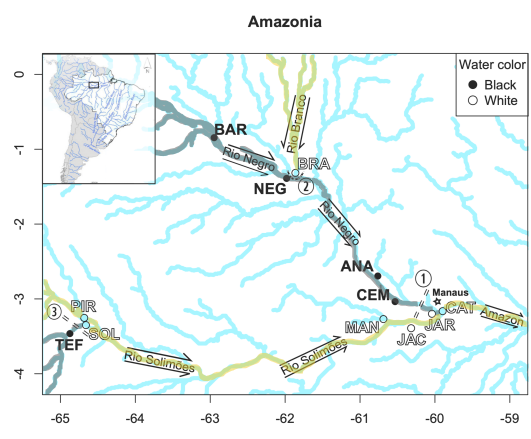

Figure 1: Location of the sampling sites $(n=12)$ in the Amazon basin. The 3 major tributaries of the Amazon are labeled, and the water flow directions are identified using arrows. Rivers of interest have been highlighted according to their water type. Water is always flowing toward the East, in the direction of the Amazon. Sites have been identified using their 3-letter acronyms and point colors are consistent with the described water type at a given site. The three genetic gaps detected in the study are also identified by numbers (1-3).

\section{Hosted file}

Table 1.docx available at https://authorea.com/users/447881/articles/546790-allopatricspeciation-rather-than-ecological-speciation-drives-evolution-in-an-amazonian-fish 


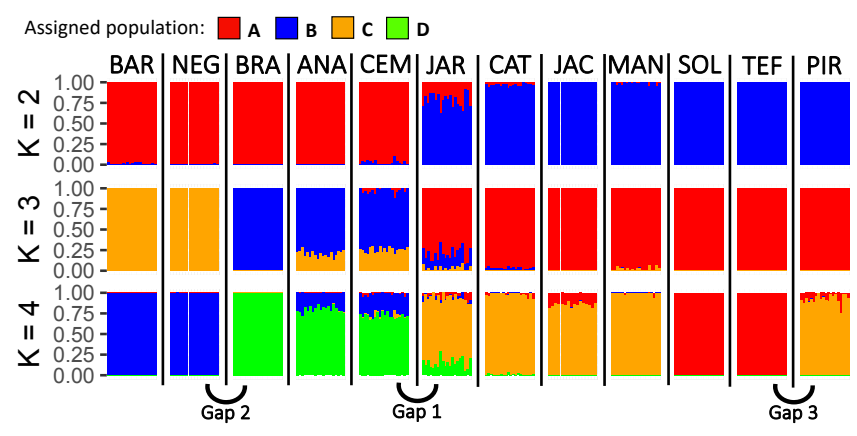

Figure 2: Posterior membership probability plots from Admixture based on the 41,268 SNPs and considering two to four genetic clusters (K). Each individual $(n=231)$ is represented by a single vertical line colored proportionally to its posterior membership probability to one of the eight genetic clusters considered for this analysis. Samples are wrapped by sampling sites. After $\mathrm{K}=4$, only one sampling site is being differentiated from the others when increasing the $\mathrm{K}$ value. The 3 genetic gaps detected in the study are identified.

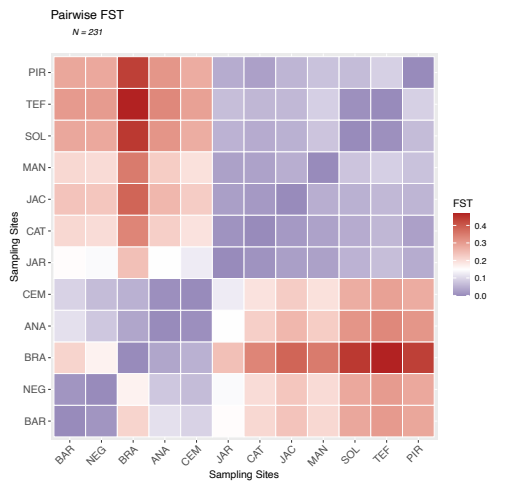

Figure 3: Pairwise Fst/( 1 -Fst) heatmap of the Fst values between the 12 sampling sites of Mesonauta festivus in the Amazon basin. Fst values were estimated using
the stamppFst function in $R$ with 100 bootstraps and assuming 4 genetic clusters. Fst values represent the genetic differentiation between each pair of sites, a value the stamppFst function in $R$ with 100 bootstraps and assuming 4 genetic clusters. Fst values represent the genetic differentiation between each pair of sites, a value
of one meaning that the populations are completely differentiated and a value of zero meaning that they are identical. Sites were ordered according to their watershed of one meaning that the populations are completely differentiated and a value of zero meaning that they are identical. Sites were ordered
of origin. For BAR, CAT, JAR, MAN, CEM, BRA, TEF, SOL and PIR $(n=20)$, for ANA $(n=19)$, and for JAC and NEG $(n=16)$. 


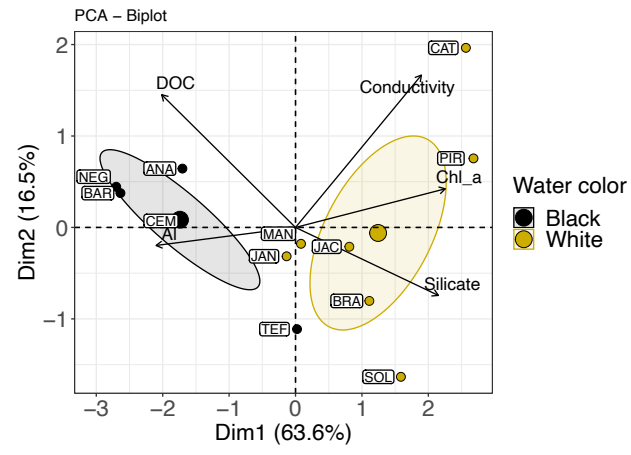

Figure 4: Biplot of the five selected environmental variables that explains most of the differences between the three water types sampled at the 12 sites of the study. Colored ellipses represent a $95 \%$ confidence interval for the two water types, with the larger circles representing the centroids (mean values) of each ellips.
The labels identify each site sampled $(n=12)$ with a three letters acronym. Vectors length represents each variable contribution to the PCA.

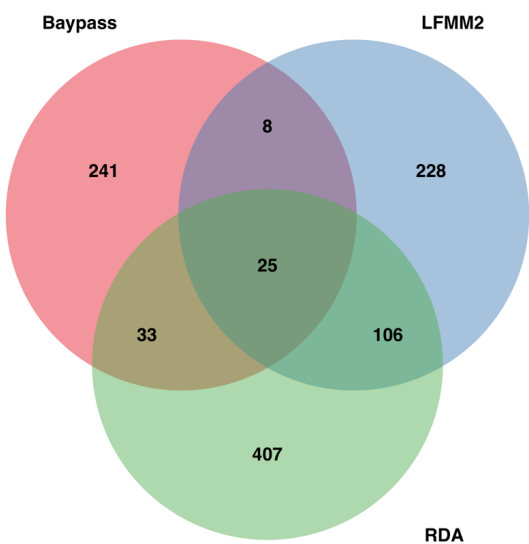

Figure 5: Venn diagram representing the number of SNPs putatively associated to environmental variables detected with each of the environmental association study methods. In total, Baypass detected 307 SNPs putatively under selection, while LFMM2 and RDA respectively detected 367 and 584 SNPs. This Venn diagram was produced using the library VennDiagram in R. 


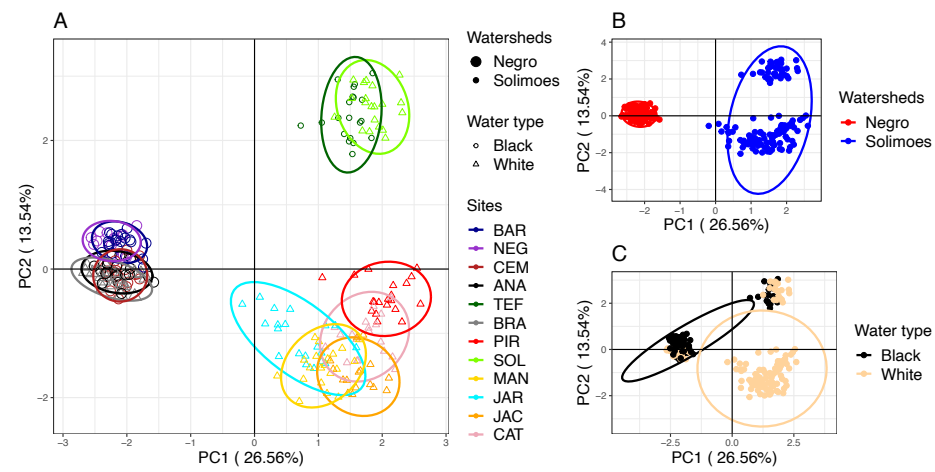

Figure 6: Principal component analysis, from black and white water sites $(12$ sites $(\mathrm{n}=231)$ ), based on the $172 \mathrm{SNPs}$ associated to water type or to environmental variables associated with diverging water types. The percentage of the variance explained by the principal component's axes are
written in parentheses. Ellipses represent the $95 \%$ confidence interval of the groupings position in space. In A, the ellipses represent the sampling site, the point size represents the site's watershed and the point shape the site's water type. In B, samples are clustered by watershed, and, in C, they are clustered by water type. See in figure S10 PCAs with the 41,268 SNPs, which shows a very similar clustering pattern. 